\section{Anmerkung}

1. Es ist erst einmal nicht recht zu erkennen, warum der BGH insgesamt Werkvertragsrecht anwenden will. Um die Vergütungspflicht für das gemietete Terminal entfallen zu lassen, reicht die typische Argumentation bei Verträgen über Hardware und Software mit einheitlichem Vertragsziel aus, daß der Anwender auch von dem für sich allein sinnlosen Vertragsteil über die Hardware zurücktreten kann. Mit der Einheit der Leistung argumentiert der BGH überzeugend unter II.3 zur Verschiebung des Liefertermins auch für die Hardware.

2. Die Anwendung von Werksvertragsrecht insgesamt zeigt, wie eng der BGH hier den Zusammenhang von Hardware und (Anwendungs) Software sieht, nämlich so, daß er nicht wie üblich nach der Theorie vorgeht, jede Leistung nach eigenem Vertragstyp zu behandeln.

3. Die Bedeutung der Anwendung von Werkvertragsrecht insgesamt liegt $\mathrm{m}$. E. in der Behandlung der Frage, welche Kapazitāt die - der Anwendungssoftware dienende - Hardware haben muß. Nach Mietoder Kaufrecht wären bestimmte Geräte zu liefern. Reicht deren Kapazität nicht aus, ist die Sache fehlerhaft. Ein Anspruch auf Aufrüstung ist nicht gegeben. So hingegen kommt es nach Werkvertragsrecht darauf an, daß „solche Kapazität zur Verfügung zu stellen“ ist, die ausreicht.

4. Welche Kapazität geschuldet ist, wird hier bei zu erstellender Software im Grundsatz sehr salopp ent- schieden: nämlich eine Reservekapazität in begrenztem Umfang - die, das sieht auch der BGH, im Einzelfall schwierig zu bestimmen ist. Aber der Grundsatz ist deutlich: Reservekapazität ist nötig. Das muß hinterfragt werden. Auszugehen ist davon, daß, wenn der Anwender hinsichtlich der Anwendungssoftware $\mathrm{Zu}$ satzwünsche hat, er diese zu bezahlen hat. (Oder geht der BGH davon aus, daß ein Pauschalpreis für die zu erstellende Software ebenfalls eine gewisse Menge an Zusatzwünschen abdeckt, weil diese vorhersehbar sind?). Lösen die Zusatzwünsche zusätzlichen Bedarf an Kapazität aus, so ist auch diese zu bezahlen. Ist die Hardware in kleinen Stufen aufrüstbar, so kann Kapazität günstig nachbeschafft werden. Der Anwender ist dann u.U. interessiert, erst einmal keine Zusatzkapazität angeboten zu bekommen. Schließlich braucht er ja nicht solche Zusatzwünsche zu haben, die in die Kapazität gehen (durchaus nicht nötig!).

Danach ist nur eine solche Reserve geschuldet, die höchstens etwa die Hälfte der nächsten Ausbaustufe ausmacht.

Die Frage, was geschuldet ist, wenn solch ein Ausbau - im Extremfall - uichr möglich ist, weist m.E. darauf hin, wie das Problem dogmatisch korrekt einzuordnen ist: Es geht $\mathrm{m}$. E. um eine Beratungspflicht seitens des Lieferanten: Er hat den Kunden auf das Problem der Reservekapazität und der möglichen Ausbanstufen hinzuweisen. Dann kann der Anwender auf Grund seiner Planungen Kapazitätsbedarf - aus Anwendersicht - prognostizieren.

\title{
Nichtlieferung der Benutzerdokumentation
}

\section{OLG Stuttgart, Urteil vom 1. Oktbber 1986 (4 U 187/85)}

\section{Nichtamtliche Leitsätze}

1. Verträge über Lieferung von DV-Systemen sind dann Werklieferungsverträge über nicht vertretbare $\mathrm{Sa}$ chen, wenn nicht nur die Ubereignung bereits fertiger Waren geschuldet wird, sondern vor allem die individuelle Anpassung der Programme (durch Einrichten) an die Besonderheiten jedes einzelnen Betriebs, die Einarbeitung des Betriebspersonals und die Erfassung der betrieblichen Daten.

2. Die Benutzerdokumentation der Programme ist wesentlicher Bestandteil der Lieferung. Die Bezahlung des Systems kann kaum als Gesamtabnahme verstanden werden, weil die Benutzerdokumentation ein wesentlicher, ohne weiteres abgrenzbarer Teil ist. Das gilt insb., wenn die Benutzerdokumentation wegen der erst nach Lieferung der Programme vorzunehmenden Anpassung der Programme erst nach Lieferung fertiggestellt werden kann.

3. Zu den Anforderungen an die Benutzerdokumentation bei Dialogprogrammen. Je komfortabler das
Programm ist, desto kürzer kann die Benutzerdokumentation sein. Die Bedienerführung im Dialog ersetzt nicht die Benutzerdokumentation.

4. Die an den Leasinggeber gerichtete Übernahmebestätigung beinhaltet keine Abnahme gegenüber dem Lieferanten. Zur Auslegung der in der Zahlung seitens des Leasinggebers liegenden Abnahmeerklärung.

5. Zur Lösung vom Vertrag gegenüber einem Lieferanten, der ein Gerät - nachträglich - zu einem DVSystem geliefert hat, wenn der Anwender vom Liefervertrag über das DV-System mit einem anderen Lieferanten zurücktritt und die beiden Lieferanten wirtschaftlich eng verbunden sind.

\section{Paragraphen}

BGB: $\S 325 ; \S 326 ; \S 631 ; \S 651$

HGB: $\S 377 ; \S 378$ 


\section{Stichworte}

Abnahme - Unterzeichnung einer Ubbernahmebestätigung bei Leasing; Benutzerdokumentation; Koppelung von Hardware und Software - zwei Lieferanten; Leasing - Übernahmebestâtigung; Lieferung eines DV-Systems - rechtliche Einordnung; Werklieferungsvertrag über eine nicht vertretbare Sache.

\section{Tatbestand}

„Die Kläger, Getränkegroßhändler, nehmen die Beklagten auf Rückabwicklung von Verträgen über Computer und Programme int Anspruch.

Im Sommer 1981 bot die Beklagte Ziff. 1, deren persönlich haftender Gasellschafter der Beklagte Ziff. 2 ist, verschiedenen Mitgliedern einer Getränke-Fachgroßhandels-Cooperation, darunter den Klägern, ein System, bestehend aus einem Computer mit Drucker, einem Getränke-Fachgroßhandels- und einem Finanzbuchhaltungsprogramm, an ...4, und zwar zu Preisen zwischen DM 18400,- und DM 22 370,- brutto.

„Der Kläger Ziff. 1 erwarb das System von der Beklagten Ziff. 1, während die Kläger Ziff. 2-5 die Systeme bei der Firma (Leasinggeber, im folgenden LG), mieteten....

Nach Auslieferung und Einarbeitung Ende 1981/ Anfang 1982 kam es bei den Klägern bei der Arbeit mit dem System immer wieder zu Schwierigkeiten, die die Kläger auf mangelhafte Programme und die Beklagten auf Bedienıngsfehler zurückführen. Am 8. März 1983 kam es auf Veranlassung des Zeugen ... zu einem Treffen.... Der Zenge (hat) ... ausgesagt, der Beklagte Ziff. 2 habe bei der Besprechung ... gesagt, das Benutzerhandbuch für die Systeme sei noch nicht fertig, es werde nachgeliefert. ..." Nach vergeblicher Nachfristsetzung mit Ablełinungsandrohung erklärten die Kläger ${ }_{n}$ mit Schreiben vom 19. Juli 1984 die Rückgängigmachung der Werklieferungsverträge.

Sie verlangten vor dem Landgericht die Rückzahlung der von ihnen (Kläger Ziff. 1) geleisteten Vergütungen ... Das LG hat die Klagen wegen Verjährung abgewiesen.

„... Bezüglich der Vertragsabwicklungen ist zwischen den Parteien unstreitig“, daß die Kläger spätestens bis April 1982 das System erhalten und daß der Kläger Ziff. 1 bzw. die LG nach Unterzeichnung der Übernahmebestätigung seitens der Kläger Ziff. 2 bis 5 die Rechnungen spätestens bis April 1982 bezahlt haben. „... Wegen Schwierigkeiten mit dem Drucker erhielt die Klägerin Ziff. 2 von einer ,Firma' XX (gleiche Adresse und Telexanschluß wie die Beklagte Ziff. 1) im Austausch einen Facit-Drucker mit Interface gegen Aufzahlung von 3842,- DM. Bei dieser ,Firma' handelt es sich um eine Gesellschaft bürgerlichen Rechts, deren einer Gesellschafter der Beklagte Ziff. 2 ist. ..."

\section{Entscheidungsgründe}

„Die Klage ist weitgehend begründet, da die Beklagte ... nach Rücktritt von den Verträgen auf Rückzahlung der erhaltenen Beträge haftet.
Mit dem Landgericht geht der Senat davon aus, daß die zwischen dem Kläger Ziff. 1 bzw. der Firma LG und der Beklagten Ziff. 1 ... abgeschlossenen Verträge Werklieferungsverträge über nicht vertretbare Sachen darstellen. Inhalt der Vereinbarungen war nämlich nicht nur die Übereignung bereits fertiger Waren, sondern vor allem auch die individuelle Anpassung der mitgelieferten Programme an die Besonderheiten jedes einzelnen Betriebs, die Einarbeitung des Betriebspersonals und die Erfassung der betrieblichen Daten. Insbesondere aus S. 2 des Angebots - alles aus einer Hand - ergibt sich, daß diese individuelle Anpassung auf den einzelnen Betrieb der entscheidende Unterschied zu anderen ,starren Programmen wie oft angeboten' ist. Dies verbietet auch die Aufgliederung in jeweils einen Kaufvertrag über die Geräte und einen Werkvertrag über die noch individuell anzupassenden Programme. Damit sind auf die abgeschlossenen Verträge über $\S 651$ Abs. 1 S. 2, 2. Alternative BGB die $\S \S 633$ bis $640 \mathrm{BGB}$ anzuwenden.

Bei allen 5 Verträgen gehörte äts wesentlicher Bestandteil zum Lieferumfang eine schriftliche Bedienungsanleitung (= Benutzerhandbuch) für die Programme, weil ohne sie die Geräte praktisch nicht nutzbar sind. Dies hat für die konkret gelieferten Systeme der Sachverständige zur Überzeugung des Senats begründet. ... haben die von der Beklagten gelieferten Programıne kdine ins Programm eingebaute Bedienerführung, die jeweils nach einem Schritt dem Bediener über den Bildschirm mitteilt, wie er weiter zu arbeiten hat, was in vielen ,Komfortabilitätsstufen' möglich ist. Für dem praktisehen Einsatz der Systeme bedeutet dies, daß ein Benutzerhandbuch um so umfassender sein muß, je weniger komforfabel das Programm ausgebant ist. Der Sachverständige hat insbesondere das Argument der Beklagten entkräftet, wegen des benutzten Dialogsystems sei ein Handbuch nicht erforderlich. Da grundsätzlich alle derartigen Systenıe im Dialogverfahren arbeiten, weil das Gerät den Bediener ,fragt', was es arbeiten soll, und dieser antwortet, hat das Dialogverfahren allein mit dem Einbau einer Bedienerführung ins Programm noch nichts zu tun. Wenn die Beklagten mit ihrem Einwand gemeint haben sollten, die Bediemıngsanleitung sei bereits im Prograttm enthalten, so daß ein Benutzerhandbuch nicht nur dünn ausfallen, sondern ganz entfallen könne, so fehlt hierzu jeglicher substantiierter Vortrag über den konkreten komfortablen Ausbau der Programme, der ausnahmsweise die Notwendigkeit einer schriftlichen Bedienungsanleitung entfallen ließ.

Da unstreitig das erforderliche und der glaubhaften Aussage des Zeugen ... zufolge am 8. März 1983 auch ausdrücklich zugesagte Benutzerhandbuch nicht geliefert wurde, stellt sich die Frage, ob das Fehlen des Benutzerhandbuchs einen wesentlichen Mangel des Systems darstellt, wie beispielsweise das OLG Frankfurt/ Main in NJW 85, 2278 annimmt, oder ob hier eine teilweise Nichterfüllung vorliegt. Nur im erstgenanıten Falle käme eine Verjährung nach $\S 638$ BGB in Frage. 
Die Unterscheidung ist nicht danach zu treffen, wie notwendig das Benutzerhandbuch für die Kläger ist, sondern danach, ob die Kläger das ganze System abgenommen haben, was das Landgericht für den Kläger wegen der Zahlung und für die anderen Kläger wegen der Übernahmebestätigung angenommen hat, oder ob allenfalls nur Teilabnahmen vorliegen. $\mathrm{Da}$ die $\mathrm{Ab}-$ nahme auch ein Willenselement enthält, ist zu fragen, ob die Beklagte die entsprechenden Erklärungen der Kläger als Abnahme des gesamten Systems verstehen durfte.

1. Als entsprechende Abnalnne beim Kläger Ziff. 1 kommt die vollständige Zahlung des berechneten Preises oder die spätere Benutzung des Systems in Frage. Bei der Bedeutung dieser keine ausdrückliche Abnahme darstellenden Tatbestände ist von Bedeutung, daß entsprechend den Darlegungen des Sachverständigen wegen der erst auf die einzelnen Kunden anzupassenden Programme eine Bedienungsanleitung eigentlich nicht von vornherein vorhanden sein konnte, sondern erst im Laufe der ersten Betriebszeit erarbeitet werden mußte. Davon sind ersichtlich die Beklagten selbst bis zum 8. März 1983 ausgegangen. Dann durften sie aber weder die sofortige Zahlung des gesanten Preises noch die spätere Benutzung der Programme als Abnahme der gesamten Leistung, sondern allenfalls als Teilabnahme verstehen. Der Senat verkennt nicht, daß auch ohne weiteres unvollständige Leistungen abgenommen werden können und des öfteren in Kenntnis der Mangelhaftigkeit auch abgenommen werden. Fehlt jedoch einem Werk ein so wesentlicher - ohne weiteres abgrenzbarer - Teil wie hler die Bedienungsmleitung, so darf der Unternehmer Tatbestände, die unter anderen Umständen die Billigung des Werkes insgesamt bedeuten können, nur dann als Abnahme des ganzen Werkes verstehen, wenn dies der Besteller eindeutig zum Ausdruck bringt.

Mangels Abnahme konnte der Kläger Ziff. 1 gegen die Beklagten nach $\$ \S 636$ Abs. 1, 634 Abs. 2 BGB vorgehen. Die Fertigstellung des Werkes, d.h. das Erstellen der Betriebsanleitung, war den Beklagten nicht unmöglich geworden, wenn sie anch nach dem Ausscheiden des betreffenden Sachbearbeiters hierfür einen größeren finanziellen Aufwand hätten treiben müssen. Mit Schreiben vom 14. Mai 1984 haben alle Kläger den Beklagten eine angemessene Frist von 6 Wochen zur Lieferung der Bedienungsanleitung gesetzt und darauf hingewiesen, daß danach Nachlieferungen abgelehnt werden. Nach Fristablauf sind sie mit Schreiben vom 19. Juli 1984 zarückgetreten. In entsprechender Anwendung von $\S 325$ Abs. 1 S. 2 BGB war dies möglich, weil das System ohne Betriebsanleitung praktisch nicht nutzbar war, wie der Sachverständige überzeugend dargestellt hat.

Der Rücktritt ist auch nicht in entsprechender Anwendung von $\$ \S 377,378 \mathrm{HGB}$ ausgeschlossen, nachdem ausweislich der Aussage des Zeugen ... der Beklagte Ziff. 2 noch am 8. März 1983 ausdrücklich die Nachlieferung der Bedienungsanleitung zugesagt hat.

2. Die Klägerin Ziff. 2 hat - wie die Kläger Ziff. 3 bis 5 - keinen Werkvertrag mit der Beklagten Ziff. 1, vielmehr einen Mietvertrag mit der Firma LG abgeschlossen, in dem die Fa. LG alle Ansprüche wegen Vertragsverletzungen der Beklagten Ziff. 1 an die Kläger Ziff. 2 bis 5 abgetreten hat.

„... Damit haben die Kläger Ziff. 2 bis 5 die Rechte der Firma LG aus den von dieser mit der Beklagten Ziff. 1 abgeschlossenen Werklieferungsvertägen, die denselben Inhalt haben wie der mit der Klägerin Ziff. 1 abgeschlossene Vertrag. Die Abnahme im Rahmen der Werklieferungsverträge war von der Firma LG eu erklären. Da sie jedoch die Systeme selbst überhaupt nicht in Empfang nuhm, mußte sie sich von ihren Mietern, den Klägern Ziff. 2 bis 5, sagen lassen, ob der Mietgegenstand in ,betriebsfähigem und einwandfreiem Zustand' übergeben wurde, um dann erst die Werkvergütung zu bezahlen. Pamit sind die Übernahmebestätigungen der Kläger Ziff. 2 bis 5, auch wenn sie den Beklagten ausgehändigt wurden, keine Abmahmeerklärungen, die etwa die Mieter in Vollmacht für die Vermieterfirma an den Lieferanten abgeben, sondern beschränken sich auf die Wirkung im Mietverhäitmis. Dies ergibt sich deutlich dadurch, daß die Firma LG als Adressat der Erklärung aufgeführt ist. Wenn die Beklagten sich diese Erklärungen bei den Kunden abholen und sie so schnell wie möglich an die Firma weiterleiten, so sind sie insoweit Boten der Kunden und übernehmen diesen Dienst im eigenen Interesse, um alsbald von der Vermieterin bezahlt zu werden. Eine Abnahmeerklärung der Firma LG könnte dann - wie beim Kläger Ziff. 1 - entweder in der Zahlung des Werklohns an die Beklagte Ziff. 1 oder darin gesehen werden, daß die Rima LG inre Mieter die Systeme benutzen ließ. Beide Möglichkeiten scheiden jedoch hier aus den gleichen Gründen wie beim Kläger Ziff. 1 aus. Mangels ausdrücklicher Abnahme durften die Beklagten, weil das unentbehrliche Benutzerhandbuch fehlte, allenfalls von einer Teilabnahme ausgehen.

Sollte die Firma LG, was nicht vorgetragen ist, die Übernahmebestảtigungen als eigene Erklärungen an die Beklagten weitergeleitet haben, so durften die Beklagten auch dies im Hinblick auf die noch fehlenden Bedienungsanleitungen nur als Teilabnahmen verstehen.

Mit dem wirksamen Rücktritt ... können daher auch die Kläger Ziff. 2 bis 5 Rückzahlungen von der Firma LG bezahlten Beträge mit Verzinsung ab Zahlung verlangen ...

Da allerdings mit dem Rücktritt vom Werkvertrag — wie bei der Wandlung - die Geschäftgrundlage des Mietvertrags weggefallen ist, können die Kläger Ziff. 2 bis 5 nicht Zahlung an sich, sondern nur Zahlung an die Firma LG verlangen. ..

3. Bei allen Klägern beträgt die Höhe der Zinsen $5 \%$, da die Werklieferungsverträge zwischen dem Kläger Ziff. 1 bzw. der Firma LG und der Beklagten Ziff. 1 beiderseitige Handelsgeschäfte darstellten und auch die Rückabwicklungsansprüche nach Vertragsrücktritt noch Ansprüche aus den - ungestalteten - Hardelsgeschäften sind. ... 
4. Die Klägerin Ziff. 2 kann darüberhinaus vom Beklagten Ziff. 2 Rückzahlung des Kaufpreises von 3842.- DM des von der ,Firma' XX gekauften Drukkers verlangen. Da der Beklagte Ziff. 2 sowohl Komplementär der Beklagten Ziff. 1 als auch Mitgesellschafter der XX-Gesellschaft ist, kann davon ausgegangen werden, daß für diese Gesellschaft wie für die Klägerin Ziff. 2 Geschäftsgrundlage für den Kauf des Druckers das Fortbestehen des Leasigvertrages mit der Firma LG war. Nachdem diese Geschäftsgrundlage durch den Rücktritt weggefallen ist, ist der Beklagte Ziff. 2 ... zur Rückzahlung des Kaufpreises gegen Rückgabe des Druckers zu verurteilen. ..."

\section{Anmerkung}

1. Ein kleines Mißverständnis des Gerichts: Das OLG Frankfurt hat die Nichtlieferung der Benutzerdokumentation als Mangel entsprechend der herrschenden Dogmatik im Mietrecht eingeordnet, die die Verzugsvorschriften ab Übergabe für nicht mehr anwendbar hält. Dank der durch dieses Mißverständnis für nötig gehaltenen Auseinandersetzung mit dem OLG Frankfurt haben wir eine überzeugende Abgrenzung zwischen Mangel und Teilverzug im Werkvertragsrecht (und im Kaufrecht) erhalten.

2. Aus den Preisen für die Systeme und aus dem Ablauf ist nahezu zwingend zu schließen, daß es bei der Anpassung der Programme um Maßnahmen des Einrichtens ging ( = Festlegen der gewünschten Varianten innerhalb des Anwendungsvorrats der Standardprogramme, siehe Zahrnt, DV-Verträge Rechtsfragen und Rechtsprechung, München 1987, Kapitel 8.4 (1), und nicht um Modifizierungen. Dieses Einrichten wird in der Rechtsprechung eher als werkvertragliche Leistung eingeordnet (Zahrnt a. a. O. Kapitel 8.4.1 (1)).

Das OLG geht einen Schritt weiter: Es nimmt einheitlich einen Werklieferungsvertrag über eine nicht vertretbare Sache an, und zwar weil es insgesamt um die Lieferung eines schlüsselfertigen Systems gehe. Das ist (wohl) die erste Entscheidung zu diesem DV-spezifischen Vertragstyp.

3. Das Urteil stellt in begrüßenswerter Weise die Bedeutung der Benutzerdokumentation bei Dialogprogrammen klar. Etwas eigenartig ist die Passage des Urteils, daß die Benutzerdokumentation erst nach dem Einrichten der Programme erstellt werden könnte. $\mathrm{Zu}$ einem generalisierten Programm gehört aus DV-technischer Sicht eine entsprechende generalisierte Bedie- nungsanweisung. Diese wird - wenn überhaupt! im Einzelfall ebenso wie das Programm angepaßt. Anders wäre der Aufwand viel zu hoch! Allem Anschein nach suchte der Lieferant eine Ausrede, warum er die Benutzerdokumentation nicht alsbald geliefert habe.

Das Urteil ist trotzdem richtig: Auch dieses Anpassen der Bedienungsanweisung und damit deren Lieferung kann erst nach dem Einrichten der Standardprogramme erfolgen.

4. Die Übernahmebestätigung richtet sich zwar nicht an den Lieferanten. Dieser muß und darf die sich anschließende Zahlung des Leasinggebers aber in dem Sinne verstehen, wie der Anwender die Übernahme bestätigt.

Folgt man der Argumentation des Gerichts (Gesamtleistung!), so umfaßt die Übernahmebestätigung nur eine sehr beschränkte Abnahme! Es stehen nach der Installation noch die Anpassung, die Einweisung und die Übernahme der Daten aus.

5. Damit stellt sich die Frage, wann der Anwender die Übernahmebestätigung unterschreiben soll: nach der Lieferung der Programme oder nach deren Einrichten? Der Leasinggeber wäre gut beraten, seinen Leasingnehmer aufzufordern, das erst zu tun, wenn der Lieferant alle Leistungen erbracht hat. Damit stellt sich das Problem, ob der Lieferant die von ihm an Vorlieferanten $\mathrm{zu}$ bezahlenden Lieferungen bis dahin finanzieren kann. Angesichts der hohen Konkursquote von DV-Lieferanten_wäre es ein gutes Regulativ, wenn ein Leasinggeber nur mit solchen Lieferanten zusammenarbeiten würde, die die späte Zahlung seitens des Leasinggebers verkraften können.

6. M.E. ist am interessantesten der Satz (unter 4.), daß der Anwender (Kläger Ziff. 2) auch vom Liefervertrag mit dem zweiten Lieferanten über den Drucker zurücktreten darf, weil der Liefervertrag und damit der Leasingvertrag über das System hinfällig geworden ist. Ein Satz reicht dem OLG; so eindeutig ist für den Senat die Rechtslage. Das ist ein Hinweis für die rechtliche Behandlung der häufigeren Situation, daß sich zwei Lieferanten in der Weise die Lieferung aufteilen, daß einer die Hardware (plus Betriebssystem) und der andere die (Anwendungs-)Software liefert: Angesichts der engen wirtschaftlichen Verflechtung der beiden Lieferanten hält das Gericht es für nicht weiter der Begründung bedürftig, daß der (hier später geschlossene) eine Vertrag den Bestand des (hier früher geschlossenen) zweiten zur Geschäftsgrundlage hat. Bei gleichzeitigem Abschluß wären sie also gegenseitige Geschäftsgrundlage.

(ch. z.) 